Gut, 1976, 17, 692-695

\title{
Stenosis of the colon in acute pancreatitis
}

\author{
W. S. J. MAIR, M. J. MCMAHON, AND J. C. GOLIGHER \\ From the University Department of Surgery, The General Infirmary, Leeds
}

SUMMARY A case is described demonstrating a stricture of the colon associated with acute pancreatitis and 20 similar cases found in the literature are reviewed. The colonic stenosis may be of short duration and largely due to oedema or more chronic with deposition of fibrous tissue. Many cases have been confused with a carcinoma both radiologically and at laparotomy. It is suggested that a conservative approach be adopted initially, resection being carried out if follow-up barium examination fails to show resolution of the stenosis.

Like syphillis, acute pancreatitis can be protean in its clinical presentation. Among its rarer complications is stenosis of the colon, and only 20 cases have been reported in the world literature since it was first described in 1927 (Forlini, 1927) and only one report of two cases (Miln and Barclay, 1952) has appeared in the British literature. This report describes an additional case in an elderly woman.

\section{Case report}

A 79 year old woman (A.S.M.) was admitted as an emergency to Leeds General Infirmary on 2 August 1975. She gave a three month history of anorexia and tiredness to which colicky upper abdominal pain and intermittent small volume vomiting had become added three weeks before admission. For two days she had also been passing frequent loose motions. For some years she had suffered from mild depression and she was known to have anaemia of unexplained aetiology.

Examination on admission showed her to be very pale, with a low grade pyrexia $\left(37 \cdot 2^{\circ} \mathrm{C}\right)$. There was some tenderness in the epigastrium and right hypochondrium. She was treated conservatively with fluids and bed rest, and by the next day abdominal tenderness had lessened and a mass could be felt in the right hypochondrium below a palpable enlarged liver.

Her haemoglobin level on admission was $5.9 \mathrm{~g} / \mathrm{dl}$. Blood and sternal marrow examination showed the anaemia to be megaloblastic in type and serum folate and vitamin $B_{12}$ estimations confirmed this but further investigation was interrupted by the need for urgent surgical intervention. Her serum amylase level on admission was within normal limits.

Received for publication 14 June 1976
Examination of faecal samples for occult blood was negative on three occasions. A barium enema was performed five days after admission in the belief that the mass was colonic in origin; it showed an irregular stricture, with mucosal destruction in the caecum and ascending colon. This was thought to be neoplastic (Figure). After adequate transfusion with blood a laparotomy was carried out.

At operation a mass was found in the upper abdomen involving the head of the pancreas, the right side of the transverse mesocolon, and the contiguous greater omentum. Omental patches of fat necrosis were clearly seen and when the lesser sac was examined the body and tail of the pancreas were found to be similarly affected. The ascending colon and hepatic flexure passed in front of this mass but were not involved. As there appeared to be no actual narrowing of the bowel apart from that produced by oedema, and as the caecum and small bowel were of normal calibre, biopsy of the mass was the only procedure carried out. Postoperative progress was uneventful and her abdominal pain, vomiting, and diarrhoea resolved. The biopsy showed features of fat necrosis only.

\section{Discussion}

Inflammatory stenosis of the colon associated with acute pancreatitis was first described by Forlini (1927) in a man who died of apparent small bowel obstruction. Including the case described in this report, the association has been recorded in an additional 20 patients since the original description (Table), though it may well occur much more commonly.

There is a male preponderance among these cases $(71 \%)$ and the average age was 53 years. It is 


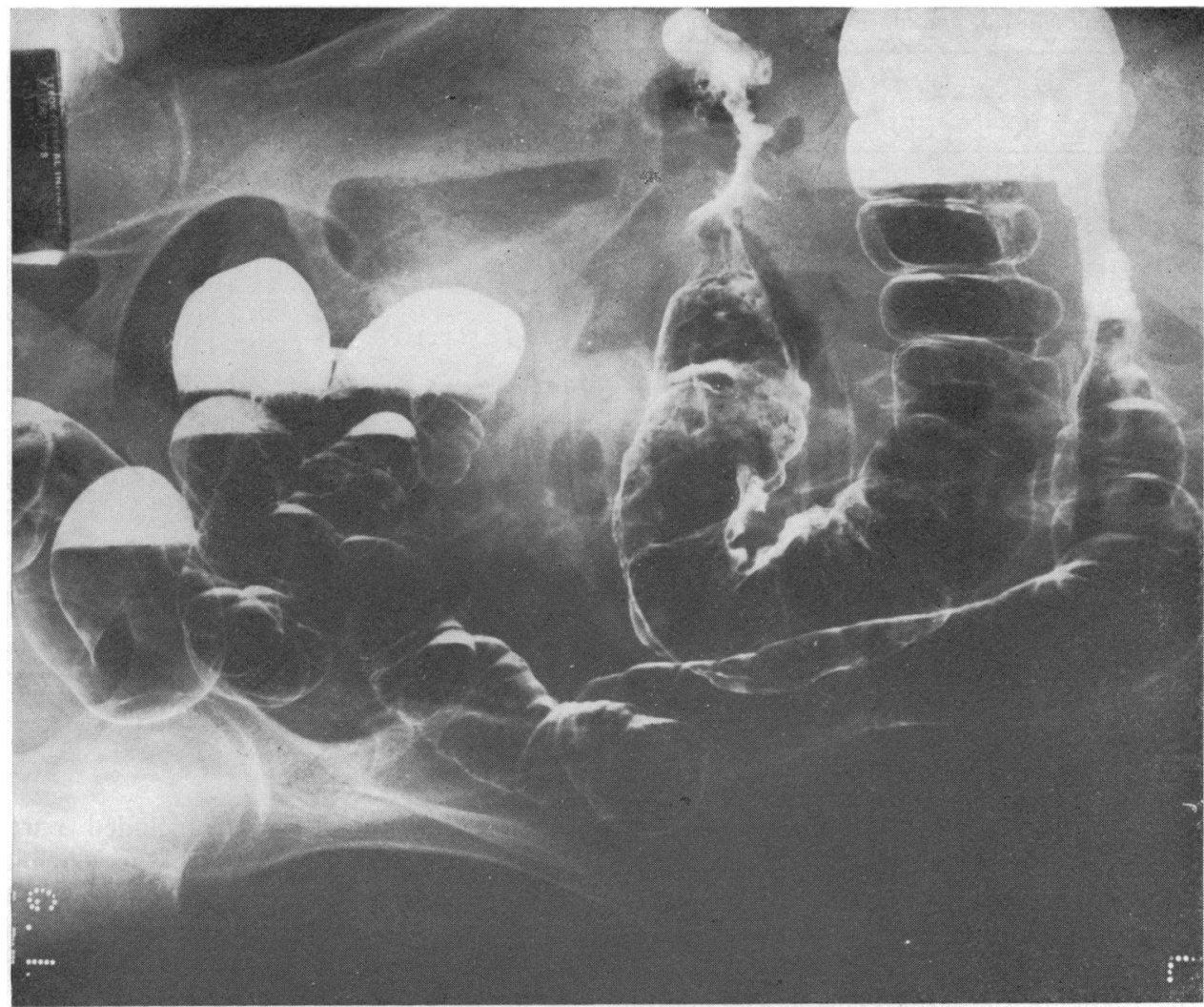

Figure Lateral beam double contrast barium enema

interesting that our patient was 79 years old and the other two cases described from Great Britain were men of 71 and 73 years (Miln and Barclay, 1952). This may be related to the somewhat unusual pattern of pancreatitis seen in Britain, where alcohol plays a minor aetiological role.

Even with full clinical details it is difficult to date the onset of the colonic problem. The figures given in the Table are estimates of the longest period of time that the stenosis is likely to have been present. The acute nature of the illness, the operative findings of an acute inflammatory reaction, and the absence of chronic changes in the colonic wall on histology indicate a very short duration (seven cases less than 10 days). The rapid return to normality in those cases where no treatment was directed to the colon itself supports this. In five cases, however (Remington et al., 1947; Mohuiddin et al., 1971; Hunt and Mildenhall, 1975; Theodoropoulos et al., 1975), there was clinical or pathological evidence that a colonic lesion had been present for some months at least. In our case, colonic involvement may have dated from the onset of pain and vomiting (three weeks) or the onset of diarrhoea (two days).

There appear to be three principal types of presentation. Most commonly (11 patients, $52 \%$ ) during an attack of acute pancreatitis some feature has drawn attention to the large bowel. The "colon cutoff' sign (Stuart, 1956) on the plain abdominal radiographs led to a barium enema in five patients $(24 \%)$. In four patients $(19 \%)$ frank rectal bleeding, the persistence of occult blood in the motions, or the appearance of a faecal fistula indicated a colonic lesion. In two cases it is unclear why a barium study was performed.

Secondly, five patients $(24 \%)$ were investigated for clinical evidence of large bowel obstruction or diarrhoea, and only at laparotomy, or in one case necropsy, was the existence of pancreatitis discovered.

Thirdly, and not so commonly in the more recent reports, is a group of five patients $(24 \%)$ who at laparotomy for an acute abdominal emergency of uncertain aetiology were found to have pancreatitis 
Table Twenty-one cases of colonic stenosis associated with acute pancreatitis

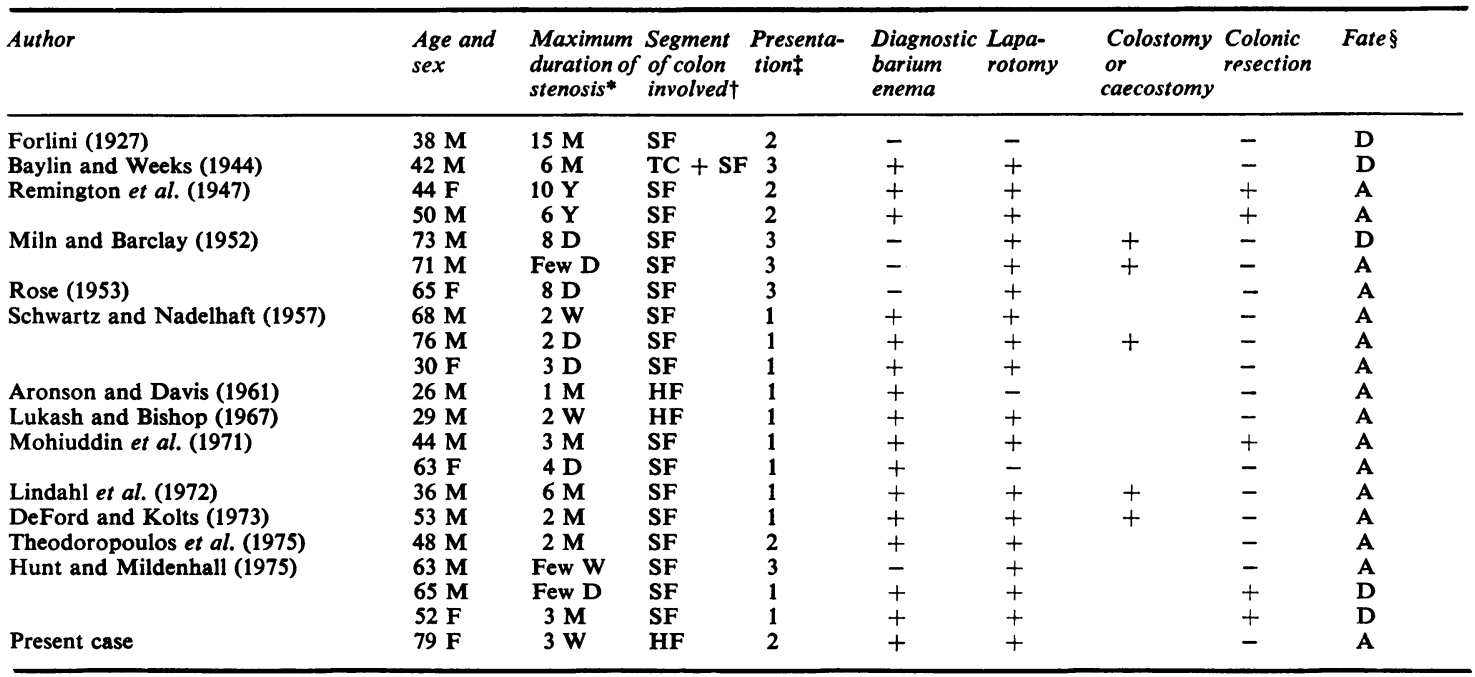

*D : day. W : week. M : month. Y : year. †HF : hepatic flexure. TC : transverse colon. SF : splenic flexure. $¥ 1:$ acute pancreatitis + indication of colonic lesion. 2 : presentation as large bowel lesion. 3 : abdominal pain and emergency laparotomy. §D : dead. A : alive.

with colonic involvement. The more widespread practice of routine measurement of the serum amylase level in patients with abdominal pain of unknown cause probably makes this type of presentation less common today.

Sixteen patients had a barium enema examination and in seven of these a diagnosis of carcinoma was made with some degree of conviction. Despite doubt about the nature of the stricture, it was pointed out that the mucosa appeared intact in three cases, though in others evidence of mucosal destruction was seen. In two cases the lesion was thought to be a carcinoma at laparotomy.

The presence of fat necrosis was noted in almost every case, indicating at least some degree of acute pancreatic inflammation. While there was widespread, fulminating pancreatitis in some cases (for example, Baylin and Weeks, 1944) in others only a localised area of pancreatic inflammation was present at the time of laparotomy. The rest of the pancreas displayed a spectrum of change from apparent normality to well-developed chronic pancreatitis.

In the acute cases variation existed in the pathology of the colonic lesion from oedematous thickening of the bowel wall to a more destructive lesion with mucosal ulceration, submucosal haemorrhage, vessel thrombosis, and muscle necrosis. Hunt and Mildenhall (1975) demonstrated submucosal fibrosis in a case of longer duration, and in the most chronic cases there was a well-developed fibrous stricture.

In some instances the colon was buried in a large inflammatory mass which included part of the pancreas (usually the tail), and from which it was not possible to separate it at laparotomy. In others the colon was found to be easily freed from the inflamed pancreas and after mobilisation it became clear that no intrinsic colonic lesion existed.

In 18 cases $(86 \%)$ the area of narrowing lay at or near the splenic flexure. While this area of the colon may be something of a weak point with respect to its blood supply (Griffiths, 1956) and has been found to be commonly involved in ischaemic colitis (Marston et al., 1966), it is difficult to escape the conviction that the high incidence of involvement of the splenic flexure area in association with pancreatitis is due to the close anatomical relationship of this part of the colon to the tail of the pancreas. Our case and two others (Aronson and Davis, 1961; Lukash and Bishop, 1967) were found to have a stenotic area at the hepatic flexure, and in the two patients who were taken to laparotomy it was evident that the stricture was related anatomically to inflammation of the head of the pancreas. A lesion of the transverse colon was found in one case only (Baylin and Weeks, 1944). The patient died of fulminating pancreatitis with large areas of fat necrosis including a stenotic, oedematous, and inflamed area at the splenic flexure.

The mortality rate for the whole series was $24 \%$ (five cases), and followed laparotomy in four cases. The two most recent deaths were consequent upon colonic resections at a time when the inflammatory process was still active. 
Laparotomy was carried out in 18 cases $(86 \%)$ and in eight of these (38\% of total series) no further procedure apart from biopsy was performed as far as the colon was concerned. There was one death among this group of eight, a patient with fulminant pancreatitis (Baylin and Weeks, 1944). In five patients a caecostomy or colostomy was performed, again with one death, but in this patient a distal pancreatectomy was also performed (Miln and Barclay, 1952).

With the exception of Forlini's original case, two patients were managed conservatively, once the clinical diagnosis became apparent. Follow-up barium enemas indicated resolution of the stenotic area.

Clearly, an awareness of the existence of colonic strictures as a complication of pancreatitis may well prevent untimely surgery in some patients. When there is reason to believe that the process is acute a good case can be made for a conservative approach with follow-up barium enema examinations. Nevertheless, surgery is probably required in patients who develop fibrous strictures, or in whom the diagnosis of carcinoma cannot be excluded, but, if possible, it should be delayed until the pancreatitis is quiescent. Should this not be possible, there is evidence that a defunctioning colostomy is of value.

\section{References}

Aronson, A. R., and Davis, D. A. (1961). Obstruction near hepatic flexure in pancreatitis: a rarely reported sign. Journal of the American Medical Association, 176, 451-452.

Baylin, G. J., and Weeks, K. D. (1944). Some roentgen aspects of pancreatic necrosis. Radiology, 42, 466-470.
DeFord, J. W., and Kolts, B. E. (1973). Stenosis of the colon secondary to pancreatitis. American Journal of Digestive Diseases, 18, 630-632.

Forlini, E. (1927). Stenosis del colon dar pancreatite. Giornale di Clinica Medica, 8, 609-620.

Griffiths, J. D. (1956). Surgical anatomy of the blood supply of the distal colon. Annals of the Royal College of Surgeons, 19, 241.

Hunt, D. R., and Mildenhall, P. (1975). Etiology of strictures of the colon associated with pancreatitis. American Journal of Digestive Diseases, 20,941-946.

Lindahl, F., Vejlsted, H., and Backer, O. G. (1972). Lesions of the colon following acute pancreatitis. Scandinavian Journal of Gastroenterology, 7, 375-378.

Lukash, W M., and Bishop, R. P. (1967). Acute pancreatitis affecting the transverse colon: report of a case. American Journal of Digestive Diseases, 12, 734-736.

Marston, A., Pheils, M. T., Thomas, M. L., and Morson, B. C. (1966). Ischaemic colitis. Gut, 7, 1-15.

Miln, D. C., and Barclay, T. H. C. (1952). Acute colonic obstruction due to pancreatitis. Lancet, 2, 168.

Mohiuddin, S., Sakiyalak, P., Gullick, H. D., and Webb, W. R. (1971). Stenosing lesions of the colon secondary to pancreatitis. Archives of Surgery, 102, 229-231.

Remington, J. H., Mayo, C. W., and Dockerty, M. B. (1947). Stenosis of the colon secondary to chronic pancreatitis. Mayo Clinic Proceedings, 22, 260-264.

Rose, T. F. (1953). Acute necrosis of the pancreas (acute haemorrhagic pancreatitis) causing and presenting as a acute colonic obstruction. Medical Journal of Australia, 1 , 147-149.

Schwartz, S., and Nadelhaft, J. (1957). Simulation of colonic obstruction at the splenic flexure by pancreatitis: roentgen features. American Journal of Roentgenology, 78, 607.

Stuart, C. (1956). Acute pancreatitis: preliminary investigation of a new radiodiagnostic sign. Journal of the Faculty of Radiologists, 8, 50-58.

Theodoropoulos, G., Archimandritis, A., and Kalos, A. (1975). Stenosis of the colon secondary to pancreatitis: report of a case. Discases of the Colon and Rectum, 18, 158-160. 